\title{
Intermediate-frequency Raman modes for the lower optical transitions of semiconducting single-walled carbon nanotubes
}

\author{
Zhengtang Luo and Fotios Papadimitrakopoulos \\ Nanomaterials Optoelectronics Laboratory, Department of Chemistry, Polymer Program, Institute of Materials Science, \\ University of Connecticut, Storrs, Connecticut 06269-3136, USA \\ Stephen K. Doorn* \\ Chemistry Division, Los Alamos National Laboratory, Los Alamos, New Mexico 87545, USA
}

(Received 18 January 2007; published 24 May 2007)

\begin{abstract}
A new class of intermediate-frequency modes (IFMs) associated with the $E_{22}^{S}$ and $E_{11}^{S}$ optical transitions of bundled HiPco single-walled carbon nanotubes (SWNTs) have been investigated via tunable laser (700$985 \mathrm{~nm}$ ) resonance Raman spectroscopy. "Steplike" dispersive behavior was observed for these IFMs, along with associated clusters of radial breathing mode (RBM) overtones at higher frequencies. While the excitation profiles of both RBM and RBM overtones follow a classical behavior predicted by resonance Raman theory, significant differences are observed for the IFM excitation profiles. The observed IFM maxima were found to obey a resonance behavior based on a combination of the $E_{22}^{S}$ and $E_{11}^{S}$ transition energies, scaled by the inverse diameter of the respective nanotube. Only IFMs for the $\bmod (n-m, 3)=2$ nanotubes are visible, with intensities found to obey the family-based chiral angle dependence similar to previously reported electron-phonon interaction patterns observed for the RBM.
\end{abstract}

DOI: 10.1103/PhysRevB.75.205438

PACS number(s): 78.67.Ch, 63.22.+m, 78.30.-j, 78.66.Tr

\section{INTRODUCTION}

Single-walled carbon nanotubes (SWNTs) can be visualized as seamless rolled-up graphite sheets, for which the Hamada vector, $\mathbf{C}_{\mathbf{h}}=n \mathbf{a}_{1}+m \mathbf{a}_{2}$ describes their chirality, where $n$ and $m$ are integers and $\mathbf{a}_{1}$ and $\mathbf{a}_{2}$ are the unit vectors on the graphene sheet. Resonance Raman spectroscopy (RRS) is one of the main structural characterization techniques in the study of SWNTs. ${ }^{1,2}$ The fundamental vibrational modes of nanotubes in RRS provide detailed information on the structure and optoelectronic properties of these quasi-onedimensional materials. In particular, the frequency dependence of the radial breathing modes (RBMs), typically observed between 100 and $400 \mathrm{~cm}^{-1}$, is inversely proportional to nanotube diameter. ${ }^{1}$ Moreover, the RBM intensities show strong resonance behavior with the optical transitions ( $E_{i i}$, index $i$ refers to the $i$ th subband of SWNTs), which can lead to an unambiguous assignment of the $(n, m)$ for a given sample, through pairing of the experimentally measured excitation maxima with the corresponding RBM frequency. ${ }^{3-5}$ On the other hand, while single nanotube spectroscopy indicates that the $G^{+} / G^{-}$intensity ratio depends on chiral angle, the frequencies of the graphitelike $\mathrm{G}\left(1500-1600 \mathrm{~cm}^{-1}\right)$ and defect-related D (1200-1400 $\left.\mathrm{cm}^{-1}\right)$ modes are only weakly dependent on the $(n, m)$ nanotube structure. ${ }^{6,7}$ At present, only limited experimental reports exist for the weak Raman modes that lie between the low (RBM) and high frequency ( $G$ and $D)$ regions, which are typically called intermediatefrequency modes (IFMs)., ${ }^{1,-11}$ Alvarez et al. have demonstrated that certain of these modes display a strong dispersive nature as a function of excitation energy. ${ }^{10}$ More recently, Fantini et al. investigated the $600-1100 \mathrm{~cm}^{-1}$ spectral region of several bundled SWNT samples using RRS with a tunable laser system in the energy range between 1.62 and $2.71 \mathrm{eV} .{ }^{12,13}$ A rich set of IFMs that exhibit steplike disper- sive behavior were identified and explained based on a process involving optical absorption and emission between the $E_{44}^{S}$ and $E_{33}^{S}$ states, mediated by two $E_{3}$ symmetry phonons (one optic and one acousticlike phonon), for $\bmod (n-m, 3)$ $=2$ (hereafter referred to as mod-2) nanotubes. Initial interpretation of the IFMs (Ref. 12) relied on theoretical $E_{44}^{S}$ and $E_{33}^{S}$ values obtained from single-particle tight-binding theory for isolated nanotubes without electron-hole interactions, ${ }^{14,15}$ which are now known to be significant. ${ }^{16,17}$ Improvements in calculated $E_{44}^{S}$ and $E_{33}^{S}$ values through use of an extended tight-binding model that accounts for both curvature and many-body effects validated the original two-phonon scattering model and extended the results to transitions in metallic nanotubes, ${ }^{13}$ yet neither approach takes into account the known environmental perturbation of transition energies due to bundling. ${ }^{18-20}$ The generalized model for the IFMs suggests similar behavior should be observable for the lower optical transitions between $E_{22}^{S}$ and $E_{11}^{S}$ as well.

In this work, we investigated the $380-650 \mathrm{~cm}^{-1}$ Raman spectral region, along with the RBM region $\left(100-380 \mathrm{~cm}^{-1}\right)$ of bundled HiPco SWNT samples using a tunable laser with energy range of 1.259 to $1.771 \mathrm{eV}$ (or 985 to $700 \mathrm{~nm}$ ), which is mainly in resonance with the $E_{22}^{S}$ and certain $E_{11}^{S}$ transitions. Steplike dispersive behavior of IFM peaks was observed, in accordance with the previous reports, along with RBM overtones that were clustered by $(2 n+m)$ families. By series deconvolutions of all the RRS spectra, we were able to extract the IFM contribution and corresponding excitation profiles for 14 individual $(n, m)$ SWNTs. Correlation of IFM behavior to simultaneously obtained RBM excitation profiles allowed accurate assignment of the IFMs to specific ( $2 n$ $+m$ ) families. The IFM excitation profile maxima can be described as a combination of the $E_{22}^{S}$ and $E_{11}^{S}$ transition energies, scaled by the inverse diameter of the respective nanotube. In addition, we observe that the IFM resonance Raman 
intensities also display the same intensity trend within a given $(2 n+m)$ family that was previously reported for RBMs. $^{21,22}$

\section{EXPERIMENTAL}

As-produced HiPco SWNTs (batch HPR 107) from Rice University were used without further purification. Aggregated/bundled SWNT samples were prepared by adding $10 \mathrm{mg}$ HiPco SWNTs to $10 \mathrm{ml}$ of isopropanol, then sonicated with a tip sonicator for $1 \mathrm{~min}$, followed by drop casting of the suspension onto a glass microscope slide. An opaque SWNT film formed upon drying. Resonance Raman spectroscopy (RRS) was performed on these samples in a backscattering configuration with tunable Ti:sapphire laser excitation from $985 \mathrm{~nm}$ to $700 \mathrm{~nm}$ in $3 \mathrm{~nm}$ steps. Excitation power was maintained at $20 \mathrm{~mW}$. Individual spectra were obtained as 5 min integrations from a back illuminated, deep depletion, charge coupled device (CCD) camera, coupled to a SPEX triple monochromator. The frequency of each carbon nanotube spectrum was calibrated at each excitation wavelength with the nonresonance Raman spectrum of 4-acetamidophenol. Intensities were corrected for instrument response using a calibration curve obtained by fitting the intensity versus excitation energy curve of 4-acetamidophenol with a third order polynomial function. All Raman spectra were taken at room temperature and ambient pressure.

\section{EXPERIMENTAL RESULTS}

Figures $1(\mathrm{a})$ and $1(\mathrm{~b})$ illustrate the excitation laser $\left(E_{\text {laser }}\right.$, from 1.259 to $1.771 \mathrm{eV}$ or 985 to $700 \mathrm{~nm}$ ) dependent Raman intensities for Raman shifts in the region of 100 to $650 \mathrm{~cm}^{-1}$ for an aggregated HiPco SWNT sample. Resonance Raman spectra obtained with $E_{\text {laser }}=1.646,1.570,1.464$, and $1.331 \mathrm{eV}$ are shown in Fig. 1(b). The intensities in the range of $380-650 \mathrm{~cm}^{-1}$ are multiplied by a factor of 10 to render the intensity of these features comparable to those lying between 100 and $380 \mathrm{~cm}^{-1}$, which mainly correspond to the radial breathing modes (RBMs) of SWNTs. The strong resonance behavior of RBM intensities allows us to assign 28 different semiconducting SWNTs that are in resonance with the first and second SWNT optical transitions $\left(E_{11}^{S}\right.$ and $\left.E_{22}^{S}\right) .{ }^{3,19}$ As indicated in Fig. 1, these RBM Raman intensities are clustered into five regions, dominated by nanotubes in the $(2 n+m=$ const $)$ families of $19,22,25,28$, and 31. Primarily nanotubes of $\bmod -2$ type [i.e., $\bmod (n-m, 3)=2]$ are observed, due to their stronger electron-phonon interaction matrix elements in comparison to their mod-1 counterparts. ${ }^{3,21-23}$ Detailed $(n, m)$ assignments and their excitation profile analysis have been widely studied elsewhere. ${ }^{3-5,19}$

Varied Raman features are also observed in the intermediate Raman shift region between 380 and $650 \mathrm{~cm}^{-1}$. Attention needs to be drawn to the range of 380 to $510 \mathrm{~cm}^{-1}$ where the intensity patterns exhibit a similar steplike dispersive behavior reported by Fantini et al. ${ }^{12,13}$ If the IFM feature peaking at $395 \mathrm{~cm}^{-1}$ is taken as an example, as excitation

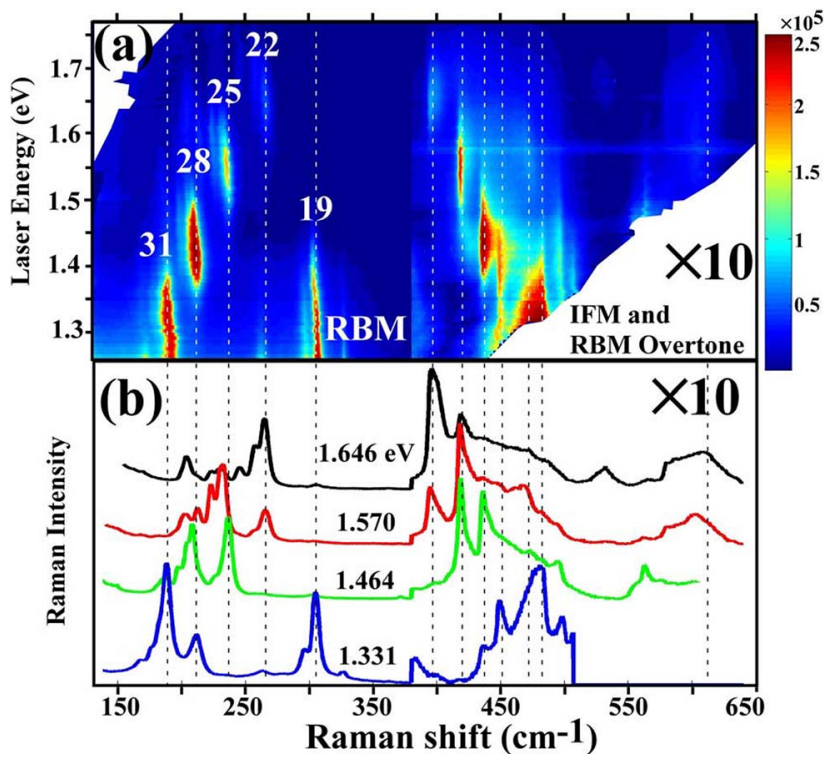

FIG. 1. (Color online) (a) Two-dimensional intensity map of laser excitation energy versus Raman shift for the radial breathing modes (RBMs), intermediate-frequency modes (IFMs), and RBM overtones for a bundled SWNT HiPco sample. RBM features are labeled according to $(2 n+m)$ families. (b) Representative four spectra obtained with $E_{\text {laser }}=1.646,1.570,1.464$, and $1.331 \mathrm{eV}$, respectively. Dashed lines are to guide the eye with respect to corresponding features in (a) and peak positions in (b). The intensities above $380 \mathrm{~cm}^{-1}$ have been multiplied by a factor of 10 in both (a) and (b) graphs to facilitate the visualization of the weak IFM and RBM overtone features, in relation to the stronger RBMs.

energy is decreased this mode loses intensity and is replaced in a single discrete jump by the IFM peaking at $419 \mathrm{~cm}^{-1}$, which in turn is replaced by a new mode at $435 \mathrm{~cm}^{-1}$ as excitation energy is further decreased. Such stepwise dispersive behavior is in contrast to the more continuous dispersion observed in double-resonance processes involving the nanotube $\mathrm{D}$ and $\mathrm{G}^{\prime}$ modes. ${ }^{2,24}$ Upon careful analysis, these peaks are asymmetric with an exception of the peak at $\sim 473 \mathrm{~cm}^{-1}$. This $\sim 473 \mathrm{~cm}^{-1}$ peak is assigned as the overtone of the RBM peak at $236 \mathrm{~cm}^{-1}$, as will be discussed later. While the peaks in the range of $460-510 \mathrm{~cm}^{-1}$ exhibit a sharp edge on the higher frequency side and a tail towards the low frequency side, the peaks in the $390-460 \mathrm{~cm}^{-1}$ range exhibit this edge on the low frequency side and tail off to higher frequencies. Such close similarity to the aforementioned reported intermediate-frequency modes (IFMs) provides strong evidence that these features should originate from a similar mechanism. Upon careful investigation, in addition to these IFM features, a number of broad and symmetric features are also visible, centered at 511,528, and $610 \mathrm{~cm}^{-1}$, in addition to the $473 \mathrm{~cm}^{-1}$ feature. The excitation ranges where these features are observed are only shifted slightly with the RBM intensity feature centered at around 236, 256, 264, $304 \mathrm{~cm}^{-1}$, respectively. The relative positions of each of the features observed in this region, and a schematic representation of their line-shape behavior, is depicted in Fig. 2(a). 

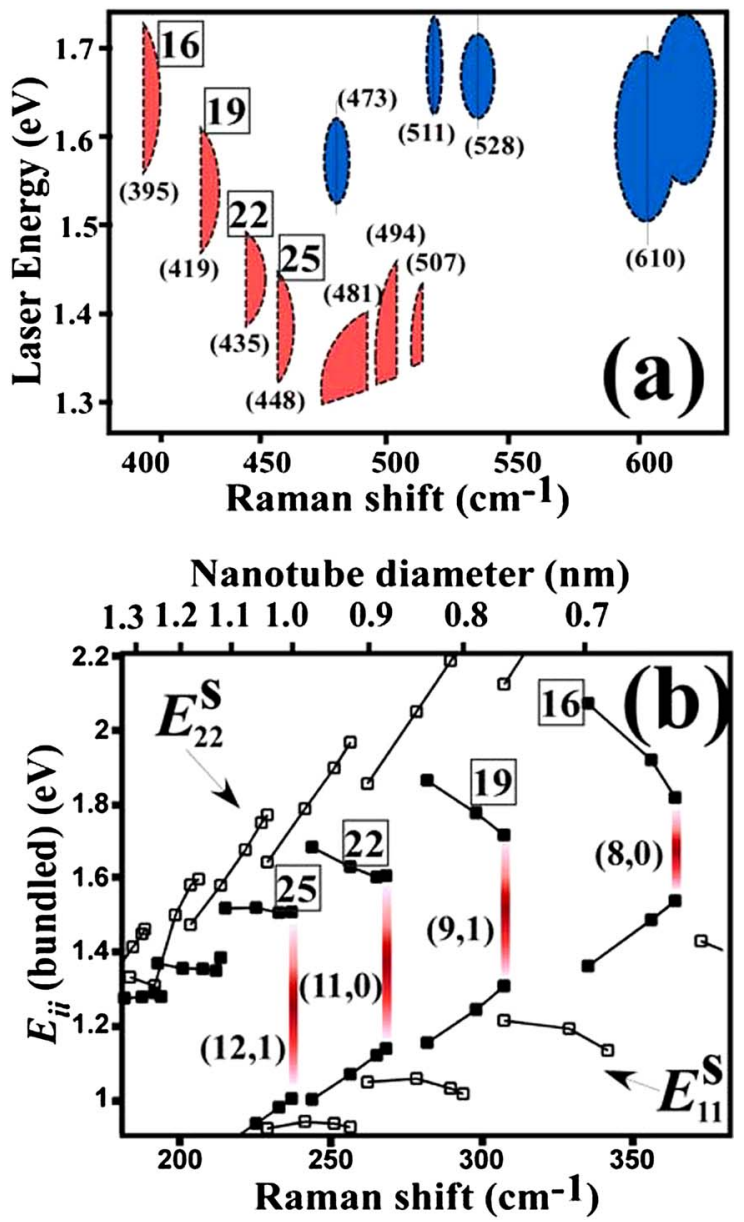

FIG. 2. (Color online) (a) Schematic mapping of the observed Raman transitions assigned to IFMs (red crescentlike features) and RBM overtones (blue ellipsoids) from Fig. 1(a). Numbers in brackets indicate the dominant peak position in $\mathrm{cm}^{-1}$. (b) Optical transition energies $\left(E_{i i}^{S}\right)$ of bundled SWNTs as a function of RBM Raman shift (bottom abscissa) and nanotube diameter (top abscissa). Scaling was based on the following relation: $\omega_{\mathrm{RBM}}=223.5 / d_{t}+12.5$ (Ref. 25). Empty and solid symbols stand for semiconducting $\bmod (n-m, 3)=1$ and $\bmod (n-m, 3)=2$ nanotubes, respectively. Curves connecting various $E_{i i}^{S}$ transitions belong to the same $(2 n$ $+m=$ const) families, depicted by the number in boxes. Vertical fading thick lines indicate the $E_{22}^{S}-E_{11}^{S}$ locale where the highest IFM intensities were observed for the zigzag or near zigzag nanotubes (shown as number pairs in brackets).

\section{DISCUSSION OF THE RESULTS}

\section{A. $(n, m)$ assignment of the Raman features}

For the RBM region of Fig. 1(a), each spectrum was deconvoluted using a summation of Lorentzian peak shapes, as discussed previously. ${ }^{21}$ Based on this, plots of the Raman cross section for each RBM frequency versus $E_{\text {laser }}$ (i.e., experimental excitation profile) are obtained. The strong enhancement observed for the RBMs when in resonance with the optical transitions, in conjunction with the $1 / d_{t} \mathrm{RBM}$ proportionality, allowed us to assign all RBM features to specific $(n, m)$ designations, in agreement with previous results. ${ }^{19}$ The $E_{i i}$ values, FWHMs $\left(\gamma_{e}\right)$, and relative Raman cross sections $[\eta(n, m)]$ for corresponding $(n, m)$ nanotubes can be obtained by fitting these experimental excitation profiles with Eq. (2) of Ref. 21. Figure 2(b) illustrates the resultant $E_{i i}$ values for our bundled SWNT samples as a function of nanotube diameter and Raman shift. These values are redshifted by about $60-100 \mathrm{meV}$ from the isolated nanotube $E_{i i}$ 's. ${ }^{4,19}$ The $E_{i i}$ values that are not found within the excitation range of our tunable laser were estimated from a $65 \mathrm{meV}$ redshifting of the photoluminescence excitation (PLE) determined values for individually dispersed SWNTs. ${ }^{19-21,25}$ The assignment of the overtone features centered at $473,511,528$, and $610 \mathrm{~cm}^{-1}$ to nanotube $(12,1)$, $(9,4)(10,2)$ and $(9,1)$ are straightforward, as they are symmetric in shape, twice the frequency of the strongest peaks in the $\mathrm{RBM}$ at 236, 256, 264, and $304 \mathrm{~cm}^{-1}$, respectively, and display similar excitation profiles (see later discussion).

In the model of Fantini et al. ${ }^{12}$ the IFM features originate from symmetry forbidden interband transitions, which in our case involve the $E_{22}^{S}$ and $E_{11}^{S}$ electronic states. In order to meet angular momentum conservation requirements, the excited electron in this process is scattered from one state to the other in a second-order process mediated by two phonons. The density of states for the involved phonons is maximized (due to momentum conservation) when $\left|k_{2}-k_{1}\right|=0$, where $k_{2}$ and $k_{1}$ describe the wave vectors for the $E_{22}^{S}$ and $E_{11}^{S}$ states, respectively. Thus, IFM intensities are expected to be greatest for $(n, m)$ nanotubes whose difference in $E_{22}^{S}$ and $E_{11}^{S}$ transition energies is minimized. As seen in Fig. 2(b), this condition is best satisfied for mod-2 nanotubes with chiral angles approaching 0 (near-zigzag structures). The IFM resonances will therefore span the gap between their associated $E_{22}^{S}$ and $E_{11}^{S}$ states, as depicted by the vertical bars in Fig. 2(b). This property provides a means for identifying the $(n, m)$ origin of a specific IFM feature. By comparing the energy range over which a particular IFM is observed [see schematic view in Fig. 2(a)] with the predicted range from Fig. 2(b), we can easily determine that the 395, 419, 435, and $448 \mathrm{~cm}^{-1}$ IFM features originate from the 16, 19, 22, and 25 $(2 n+m)$ families, respectively.

Although the near-zigzag structures will be the strongest contributors to the IFM features, the other $(n, m)$ chiralities within a specific $(2 n+m)$ family are also able to contribute to this behavior. Each asymmetric IFM feature is therefore the convolution of individual contributions from each chirality within a given $(2 n+m)$ family. To obtain the relative contribution to the IFM features from individual $(n, m)$ SWNTs, we have deconvoluted each IFM Raman spectrum using a set of Lorentzian functions, as shown in the bottom left inset of Fig. 3. In this deconvolution, we utilized a single value for the full width at half maximum (FWHM) for all contributions from mod-2 nanotubes to the corresponding IFM spectrum for each excitation line. In particular, the inset of Fig. 3 shows the two major features corresponding to the zigzag $(8,0)$ and near-zigzag $(9,1)$ nanotubes, along with progressively lesser contributions of higher chirality $(7,2)$ and $(6,4)$, as well as $(8,3)$ and $(7,5)$, respectively. In order to achieve convergence of the fitting for off-resonance peaks, their peak centers are fixed at the frequency obtained in the resonance conditions. Thus, the deconvolution procedure is self- 


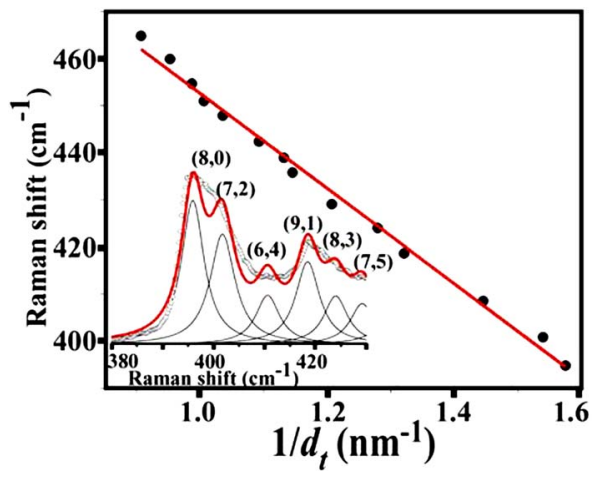

FIG. 3. (Color online) IFM Raman shifts as a function of inverse carbon nanotube diameter. The solid line is a linear fit. Bottom left inset illustrates a representative spectrum with $E_{\text {laser }}$ $=1.644 \mathrm{eV}$ (or $754 \mathrm{~nm}$ ) and the deconvolution to its constituent peaks. Experimental data is shown as open circles, with the solid line being the combined fitted spectrum.

consistent across the full excitation energy range. We find that the $5-7 \mathrm{~cm}^{-1}$ FWHM values used to deconvolute the IFMs are similiar to the approximately $7 \mathrm{~cm}^{-1}$ FWHM values of the RBMs.

\section{B. IFM phonon structures}

Deconvolution of the IFMs into the contributions from their individual $(n, m)$ constituents allows a more detailed analysis of the phonon structure that makes up the observed IFMs. As stated in the previous section, the IFMs can be related to the combination of two phonons that originated from a zone-folding procedure of two two-dimensional (2D) phonon branches, one optical and one acousticlike phonon. ${ }^{12}$ In the aforementioned RRS studies using high energy excitation laser lines, their origin was explained based on a process that involves optical absorption/emission in $E_{44}^{S}-E_{33}^{S}$ for semiconducting nanotubes. These transitions were mediated by two $E_{3}$ symmetry phonons for these semiconducting nanotubes, while for metallic $E_{11}^{M-}-E_{11}^{M+}$ transitions, this was mediated by two $E_{2}$ symmetry phonons. ${ }^{9}$ In our case of $E_{22^{-}}$ $E_{11}^{S}$ semiconducting transitions, two phonons of $E_{1}$ symmetry are required. Two phonons are required in a second-order scattering process because phonons of $E_{n}$ symmetry are not allowed in normal first-order processes due to Raman selection rules.

The Raman shift of the IFM peaks can be described by the following equation: ${ }^{12}$

$$
\omega_{\mathrm{IFM}}^{ \pm}=\omega_{O}^{ \pm} \pm \omega_{A},
$$

where $\omega_{O}$ and $\omega_{A}$ correspond to the frequencies of the optical phonon and acousticlike phonon, respectively. The plus and minus signs refer to creation and annihilation of the acousticlike phonon, respectively, with creation of an optical phonon. The negative dispersion of the modes analyzed here indicates that they correspond to the annihilation branch of Eq. (1). The frequency of the acousticlike phonon can be written as $\omega_{A}=\nu_{A} q_{\perp}$ where $\nu_{A}$ is the dispersion of the acousticlike phonon, and $q_{\perp}$ is the wave vector perpendicular to the nanotube

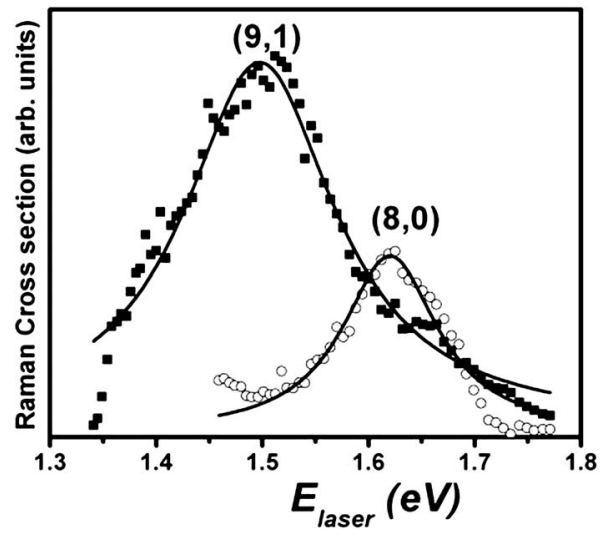

FIG. 4. Experimentally obtained IFM relative Raman cross sections (shown with " $\square$ " and " $\bigcirc$ " symbols) for the $(9,1)$ and $(8,0)$ nanotubes, respectively. Solid lines are the corresponding Lorentzian fits.

axis. ${ }^{12}$ For our case of an $E_{1}$ symmetry mode, the $q_{\perp}=2 / d_{t}$. Figure 3 depicts the IFM Raman shift plotted as a function of inverse diameter. The diameter of SWNT with structure $(n, m)$ is calculated as $a / \pi \sqrt{n^{2}+m^{2}+n m}$, where $a$ $=0.246 \mathrm{~nm}$ is the lattice constant of graphite. The dispersion is well fit by a straight line with intercept at $553.6 \mathrm{~cm}^{-1}$ and a slope of $101.2 \mathrm{~nm} \mathrm{~cm}^{-1}$. Consequently, the value of $\omega_{O}$ is seen to be $553.6 \mathrm{~cm}^{-1}$. This optical phonon energy is close to that of the $540 \mathrm{~cm}^{-1}$ phonon responsible for creation of the set of higher frequency (600-900 $\mathrm{cm}^{-1}$ region) IFM lines in the higher Raman shift region $\left(600-900 \mathrm{~cm}^{-1}\right)$ observed for electric-arc produced SWNT bundles. ${ }^{13}$ The slope of the line can be equated to $2 \nu_{A}$, which can be translated to a $\nu_{A}$ value of $1500 \mathrm{~m} / \mathrm{s}$, in agreement with the value previously found, ${ }^{12}$ and in alignment with the expected in-plane sound velocity for graphite. ${ }^{26,27}$ The good agreement of these results with the previous work of Fantini et al. ${ }^{12,13}$ provides strong evidence that the steplike behavior observed here has the same origins as the IFMs of the higher energy optical transitions.

\section{Excitation profiles of the observed IFMs}

The deconvolution of the IFM features into their individual constituents also allows plotting of the IFM relative Raman cross sections as a function of laser excitation energy $\left(E_{\text {laser }}\right)$ for each contributing $(n, m)$ structure, as shown in Fig. 4 for the $(8,0)$ and $(9,1)$ chiralities. Such curves are typical examples of the resonance excitation profiles obtained for the range of observed IFMs. Well-resolved curves were obtained for 15 different $(n, m)$ nanotubes. In most cases, such excitation profiles exhibit a symmetric quasiLorentzian line shape. No correction for the population differences of the various nanotube diameters present in the sample was performed at this stage. Interestingly, highly resolved contributions to the IFM features can be seen for nanotube species that are not typically observable in either PLE or tunable Raman RBM analysis. For example, the $(8,0)$ nanotube is not observed in PLE measurements due to its low quantum yield, nor in Raman RBM measurements since 
its Raman frequency and that of its neighbors are too close for confident deconvolution. The unique ability to observe zigzag and near-zigzag structures through the IFM features renders it a useful complementary technique for mapping the $(n, m)$ of nanotubes with low chiral angle.

It is clear from Figs. 1 and 2 that the excitation behavior of the IFMs is different from that of the associated RBMs and their overtones. This difference is compared more quantitatively for the $(12,1)$ nanotube in Fig. 5. The peak positions of the RBM and RBM-overtone profiles may be directly related to the $(12,1) \quad E_{22}^{S}$ transition energy in combination with the relevant RBM phonon energy. The
RBM resonance Raman cross section is given by the following equation: ${ }^{28,29}$

$$
\eta_{\mathrm{RBM}} \propto\left|\frac{1}{\left[E_{\mathrm{laser}}-E_{i i}-i \gamma_{e}\right]\left[E_{\mathrm{laser}}-E_{\mathrm{ph}}-E_{i i}-i \gamma_{e}\right]}\right|^{2},
$$

where $E_{\mathrm{ph}}$ represents energy of the corresponding RBM phonon and $\gamma_{e}$ is related to the lifetime of the excited electron.

Similarly, the RBM-overtone cross section can be described by the following equation: ${ }^{28,30}$

$$
\eta_{\mathrm{RBM}-\text { overtone }} \propto\left|\frac{1}{\left[E_{\mathrm{laser}}-E_{i i}-i \gamma_{e}\right]\left[E_{\mathrm{laser}}-E_{\mathrm{ph}}-E_{i i}-i \gamma_{e}\right]\left[E_{\mathrm{laser}}-2 E_{\mathrm{ph}}-E_{i i}-i \gamma_{e}\right]}\right|^{2} .
$$

According to the Raman scattering theory, the RBM excitation profile maxima should have peaks at $E_{i i}$ and $E_{i i}$ $+E_{\mathrm{ph}}$, while the overtone profiles should exhibit three peaks at $E_{i i}, E_{i i}+E_{\mathrm{ph}}$, and $E_{i i}+2 E_{\mathrm{ph}}$, where $E_{\mathrm{ph}}$ is the energy of the RBM phonon. Due to the natural linewidth resulting from $\gamma_{e}$, it is difficult to resolve this substructure due to the small value of $E_{\mathrm{ph}}(10-40 \mathrm{meV})$. The apparent excitation maxima for the RBM fundamental and overtone will therefore appear at $E_{i i}+1 / 2 E_{\mathrm{ph}}$ and at $E_{i i}+E_{\mathrm{ph}}$, respectively. Thus, the difference in energy of the two excitation peaks is expected to be on the order of one RBM phonon quantum.

Figure 5 depicts the excitation profiles of the RBM, RBM overtone, and IFM for the near-zigzag nanotube $(12,1)$. Fits to the experimental profiles are approximated by a Lorentzian line shape and used to determine the true excitation maxima. We note that a Lorentzian line shape is used as an

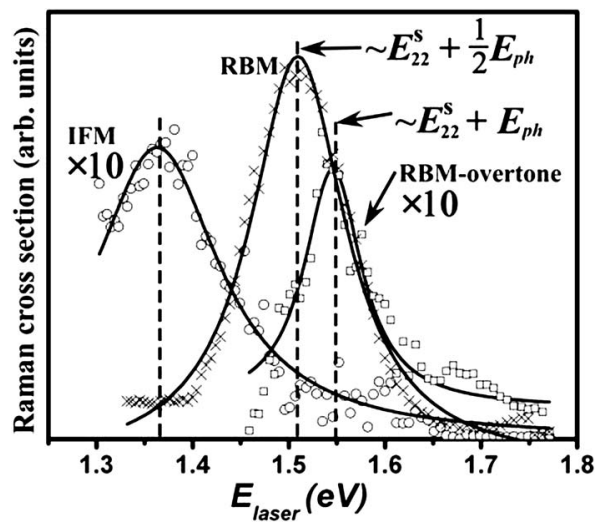

FIG. 5. Excitation profile of the IFM $(\bigcirc), \mathrm{RBM}(\times)$, and RBM overtone $(\square)$ for the $(12,1)$ SWNT. The solid curves are the Lorentzian fits. The strongest resonant positions for both RBM and RBM overtone appear to coincide with $\left(E_{22}^{S}+1 / 2 E_{\mathrm{ph}}\right)$ and $\left(E_{22}^{S}+E_{\mathrm{ph}}\right)$, respectively. The Raman cross sections for IFM and RBM overtones have been multiplied by a factor of 10 to better illustrate these features. approximation to Eqs. (2) and (3) in order to make a more direct comparison to the results of fitting to the IFM line shapes, for which no comparable Raman band-shape theory currently exists. The shift between RBM and RBM overtone excitation profiles is reflected in the difference of the RBM fundamental energy maximum $(1.509 \mathrm{eV})$ compared to that of the RBM overtone $(1.546 \mathrm{eV})$. An energy difference of $37 \mathrm{meV}$ is obtained, which is close to the energy of the RBM phonon for the $(12,1)$ tube $\left(29.4 \mathrm{meV}\right.$ or $\left.237 \mathrm{~cm}^{-1}\right)$. In contrast, the shift of maxima of the IFM excitation profile $(-146 \mathrm{meV})$ is considerably larger (and in the opposite direction) than the energy of the $(12,1)$ IFM phonon $(55.5 \mathrm{meV}$ or $448 \mathrm{~cm}^{-1}$ ). Such a distinct difference in IFM behavior, in comparison to that of the RBM and RBM overtones, in conjunction with the aforementioned distinguished peak shape, makes it clear that the IFMs are fundamentally different from other combination modes and supports the previous conclusions $^{12,13}$ that they are not likely to be a direct phonon branch of the individual $E_{i i}$. Excitation profiles were obtained for all observed IFMs. Simple Lorentzian fits have been made to these profiles to obtain the energy maxima $\left(E_{\mathrm{IFM}}\right)$ and bandwidths $\left(\gamma_{\mathrm{IFM}}\right)$ for each observed transition. Figure 6(a) shows the obtained $E_{\mathrm{IFM}}$ as a function of inverse diameter, in comparison with their $E_{11}^{S}$ and $E_{22}^{S}$ values in the aggregated sample. As yet there exists no defined theoretical basis for determining $E_{\mathrm{IFM}}$. We propose here an empirical model for calculating $E_{\mathrm{IFM}}$ from a diameter-dependent function based on weighted contributions of the individual $E_{i i}$. For this, we fit the $E_{\mathrm{IFM}}$ values with a simple function using

$$
E_{\mathrm{IFM}}=\alpha E_{22}^{S}+(1-\alpha) E_{11}^{S}
$$

An optimum fit can be obtained with $\alpha=1.41-0.7 / d_{t}$. Values of $\alpha$ were obtained in the range of 0.31 to 0.79 with a mean value at 0.58 . The calculated $E_{\mathrm{IFM}}$ values thus obtained are also plotted in Fig. 6(a) for comparison with the experimental results. 

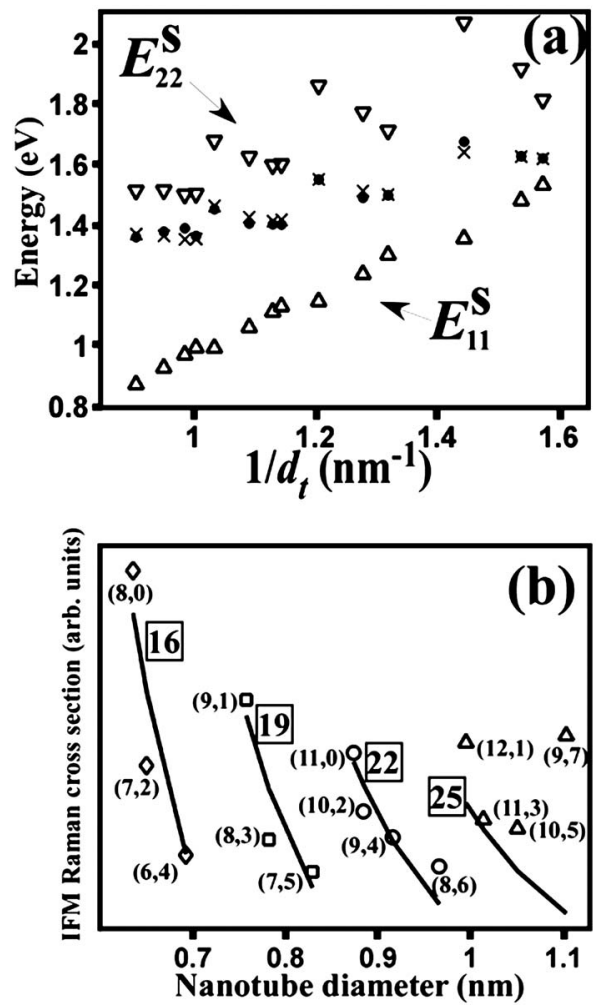

FIG. 6. (a) $E_{\mathrm{IFM}}(\bigcirc)$ as a function of inverse diameter. $E_{11}^{S}(\triangle)$ and $E_{22}^{S}(\nabla)$ are also plotted for comparison, along with the calculated $(\times)$ IFM excitation profile maxima based on Eq. (4). (b) IFM resonance Raman cross sections as a function of nanotube diameter for different $(n, m)$ SWNTs. Nanotubes belonging to the same $(2 n$ $+m$ ) families (indicated by the number in squares) are plotted using the same symbol. Solid lines represent the fit using Eq. (5).

While our analysis of the IFM data can be performed successfully within the theoretical framework of Fantini et al., recognition of the excitonic nature of the nanotube optical transitions ${ }^{14-17}$ may be required to develop a first principles analytical expression that describes the behavior of Eq. (4). This raises the possibility that the resonant scattering process we observe couples the electronic ground state to formally forbidden excitonic states intermediate in energy between the $E_{11}$ and $E_{22}$ transitions. Thus, resonance Raman mapping of the IFM excitation profiles could potentially provide an alternative optical approach to mapping the energies of such intermediate excitonic states. A theoretical investigation is underway to perform a more careful analysis of the exact phonon modes involved in the IFM process, and how they may couple to these intermediate excitonic states.

\section{IFM Raman intensities}

RBM Raman intensities have been found to be strongly dependent on family $[(2 n+m)=$ const $]$ and modality $[\bmod (n-m, 3)=1 \text { or } 2]^{3,5,21}$ These intensity variations were attributed to chirality-dependent variations in the Raman electron-phonon coupling matrix elements. ${ }^{21-23,31-34}$ By fitting the IFM excitation profiles for each $(n, m)$-SWNT using a Lorentzian line shape [shown in Fig. 4 for the $(8,0)$ and
$(9,1)$ chiralities as examples], the relative IFM resonance Raman cross section values for each $(n, m)$ nanotube can be obtained as the maximum intensity in their excitation profiles.

Figure 6(b) depicts these relative resonance Raman cross section $\left[\eta_{\mathrm{IMF}}(n, m)\right]$ values for the IFMs, plotted as a function of nanotube diameter. Cross sections have been corrected here for relative species abundance, which obeys a lognormal distribution dependent on nanotube diameter, with $\mu=0.937 \mathrm{~nm}$ and $\ln \sigma=0.199$, as was reported recently based on photoluminescence measurements. ${ }^{35}$ The geometrical patterns for the $16,19,22$, and $25 \operatorname{SWNT}(2 n+m)$ families are clearly shown [with all belonging to $\bmod (n-m, 3)$ $=2(\bmod -2)$ families $]$. Of particular note is the strong chiral angle dependence of the intensities within a given family. Intensity is found to be greatest for the near-zigzag chiralities, and falls off rapidly for larger chiral angles. This pattern matches surprisingly well with the experimentally ${ }^{21,22}$ and theoretically ${ }^{22,23,31-34}$ obtained electron-phonon interaction pattern observed for coupling of the RBM to the $E_{11}$ and $E_{22}$ transitions. To model the trends shown in Fig. 6(b), Eq. (5) was employed to account for both nanotube diameter $\left(d_{t}\right)$ and chiral angle $\left(\theta\right.$, calculated by $\theta(n, m)=\tan ^{-1}[\sqrt{3} n /(n$ $+2 m)]$ ) dependent curvature effects

$$
\eta_{\mathrm{IFM}}(n, m)=\frac{A+B \cos (3 \theta)}{d_{t}^{2}} .
$$

Here, $A$ and $B$ are two adjustable fitting factors. This empirical equation was chosen since it describes adequately well both trigonal warping effects ${ }^{36}$ and PL-derived $E_{i i}(n, m)$ values. ${ }^{25}$ Values of $A=-3.104 \times 10^{3}$ and $B=-3.9443 \times 10^{4}$ provided the best fit. The relatively high value for $B$ indicates the strong dependence of the Raman cross section on chiral angle. The large deviation between the predicted and experimental values of $\eta_{\mathrm{IFM}}(n, m)$ for larger diameter SWNTs may originate from low signal/noise ratio of the Raman spectra in this IFM region, due to their lower intensity and overlap with the RBM overtones of the $2 n+m=28$ and 29 SWNT families.

We also note the difference in intensities observed for the near-zigzag "parent" chirality for each of the $2 n+m$ families. As discussed earlier, due to momentum matching conditions, IFM intensity is expected to be greatest when the $E_{22}^{S}$ and $E_{11}^{S}$ energies are closest. The difference in these energies is ordered in the following way for the observed families: 16 $<19<22<25$. Correspondingly, Fig. 6(b) shows that the observed chirality trends of the relative Raman cross sections for these families agree with that scenario. These results suggest that the observed relative intensities result from a combination of behavior induced by momentum matching requirements and by chirality dependence in the electronphonon coupling similar to that observed for coupling to the RBM.

\section{CONCLUSION}

In summary, we have extended the generalized description of the steplike dispersive behavior of the intermediate- 
frequency modes (IFMs) to include a new class associated with the $E_{11}^{S}$ and $E_{22}^{S}$ optical transitions of bundled singlewalled carbon nanotubes (SWNTs). These results were obtained by using near-infrared tunable laser resonance Raman spectroscopy (RRS) and by correlating the IFM behavior with simultaneously obtained RBM spectra. Significant differences in the IFM excitation profiles are observed when compared to the one-phonon and two-phonon Raman processes in the vicinity of $E_{22}^{S}$. Moreover, we observe that the resonance Raman intensities also obey family patterns which can be fit using an empirical equation based on trigonal warping effects that were developed previously. ${ }^{21}$ This intermediate-frequency mode mapping methodology significantly extends the use of tunable Raman spectroscopy for nanotubes whose RBMs are off resonance with available laser lines. Probing of IFM behavior is also an important extension of techniques for characterization of low chiral angle (zigzag and near-zigzag) nanotubes, which may otherwise be thorny to tackle. Such nanotube metrology is important to guide efforts in chirality specific production and sorting.

\section{ACKNOWLEDGMENTS}

The authors would like to thank Ado Jorio for valuable discussions. Financial support from AFOSR F49620-01-10545, and ARODAAD-19-02-1-0381 are greatly appreciated (Z.L. and F.P.). S.K.D. acknowledges support of the LANL LDRD program.
*Author to whom correspondence should be addressed. Electronic address: skdoorn@lanl.gov

${ }^{1}$ A. M. Rao, E. Richter, S. Bandow, B. Chase, P. C. Eklund, K. A. Williams, S. Fang, K. R. Subbaswamy, M. Menon, A. Thess, R. E. Smalley, G. Dresselhaus, and M. S. Dresselhaus, Science 275, 187 (1997).

${ }^{2}$ M. S. Dresselhaus, G. Dresselhaus, A. Jorio, A. G. Souza Filho, and R. Saito, Carbon 40, 2043 (2002).

${ }^{3}$ S. K. Doorn, D. A. Heller, P. W. Barone, M. L. Usrey, and M. S. Strano, Appl. Phys. A: Mater. Sci. Process. A78, 1147 (2004).

${ }^{4}$ C. Fantini, A. Jorio, M. Souza, M. S. Strano, M. S. Dresselhaus, and M. A. Pimenta, Phys. Rev. Lett. 93, 147406 (2004).

${ }^{5}$ H. Telg, J. Maultzsch, S. Reich, F. Hennrich, and C. Thomsen, Phys. Rev. Lett. 93, 177401 (2004).

${ }^{6}$ A. Jorio, A. G. Souza Filho, G. Dresselhaus, M. S. Dresselhaus, A. K. Swan, M. S. Unlu, B. B. Goldberg, M. A. Pimenta, J. H. Hafner, C. M. Lieber, and R. Saito, Phys. Rev. B 65, 155412 (2002).

${ }^{7}$ M. A. Pimenta, A. Jorio, S. D. M. Brown, A. G. Souza Filho, G. Dresselhaus, J. H. Hafner, C. M. Lieber, R. Saito, and M. S. Dresselhaus, Phys. Rev. B 64, 041401(R) (2001).

${ }^{8}$ M. L. De La Chapelle, S. Lefrant, C. Journet, W. Maser, P. Bernier, and A. Loiseau, Carbon 36, 705 (1998).

${ }^{9}$ E. Anglaret, N. Bendiab, T. Guillard, C. Journet, G. Flamant, D. Laplaze, P. Bernier, and J.-L. Sauvajol, Carbon 36, 1815 (1998).

${ }^{10}$ L. Alvarez, A. Righi, T. Guillard, S. Rols, E. Anglaret, D. Laplaze, and J. L. Sauvajol, Chem. Phys. Lett. 316, 186 (2000).

${ }^{11}$ C. Journet, W. K. Maser, P. Bernier, A. Loiseau, M. Lamy de la Chapells, S. Lefrant, P. Deniard, R. Lee, and J. E. Fischer, Nature (London) 388, 756 (1997).

${ }^{12}$ C. Fantini, A. Jorio, M. Souza, L. O. Ladeira, A. G. Souza Filho, R. Saito, G. G. Samsonidze, G. Dresselhaus, M. S. Dresselhaus, and M. A. Pimenta, Phys. Rev. Lett. 93, 087401 (2004).

${ }^{13}$ C. Fantini, A. Jorio, M. Souza, R. Saito, G. G. Samsonidze, M. S. Dresselhaus, and M. A. Pimenta, Phys. Rev. B 72, 085446 (2005).

${ }^{14}$ T. Ando, J. Phys. Soc. Jpn. 66, 1066 (1997).

${ }^{15}$ C. L. Kane and E. J. Mele, Phys. Rev. Lett. 90, 207401 (2003).

${ }^{16}$ F. Wang, G. Dukovic, L. E. Brus, and T. F. Heinz, Science 308, 838 (2005).

${ }^{17}$ J. Chen, V. Perebeinos, M. Freitag, J. Tsang, Q. Fu, J. Liu, and P.
Avouris, Science 310, 1171 (2005).

${ }^{18}$ S. Reich, C. Thomsen, and P. Ordejon, Phys. Rev. B 65, 155411 (2002).

${ }^{19}$ M. J. O'Connell, S. Sivaram, and S. K. Doorn, Phys. Rev. B 69, 235415 (2004).

${ }^{20}$ Z. Luo, R. Li, S. N. Kim, and F. Papadimitrakopoulos, Phys. Rev. B 70, 245429 (2004).

${ }^{21}$ Z. Luo, F. Papadimitrakopoulos, and S. K. Doorn, Appl. Phys. Lett. 88, 073110 (2006).

${ }^{22}$ S. V. Goupalov, B. C. Satishkumar, and S. K. Doorn, Phys. Rev. B 73, 115401 (2006).

${ }^{23}$ S. V. Goupalov, Phys. Rev. B 71, 153404 (2005).

${ }^{24}$ M. A. Pimenta, E. B. Hanlon, A. Marucci, P. Corio, S. D. M. Brown, S. A. Empedocles, M. G. Bawendi, G. Dresselhaus, and M. S. Dresselhaus, Braz. J. Phys. 30, 423 (2000).

${ }^{25}$ S. M. Bachilo, M. S. Strano, C. Kittrell, R. H. Hauge, R. E. Smalley, and R. B. Weisman, Science 298, 2361 (2002).

${ }^{26}$ R. Saito, G. Dresselhaus, and M. S. Dresselhaus, Physical Properties of Carbon Nanotubes (Imperial College Press, London, 1998).

${ }^{27}$ S. Reich, C. Thomsen, and J. Maultzsch, Carbon Nanotubes: Basic Concepts and Physical Properties (Wiley-VCH, Weinheim, 2004).

${ }^{28}$ R. M. Martin and L. M. Falicov, Top. Appl. Phys. 879 (1975).

${ }^{29}$ A. Kukovecz, C. Kramberger, V. Georgakilas, M. Prato, and H. Kuzmany, Eur. Phys. J. B 28, 223 (2002).

${ }^{30}$ W. L. Peticolas, L. Chinsky, P. Y. Turpin, and A. Laigle, J. Chem. Phys. 78, 656 (1983).

${ }^{31}$ M. Machon, S. Reich, H. Telg, J. Maultzsch, P. Ordejon, and C. Thomsen, Phys. Rev. B 71, 035416 (2005).

${ }^{32}$ J. Jiang, R. Saito, A. Gruneis, S. G. Chou, G. G. Samsonidze, A. Jorio, G. Dresselhaus, and M. S. Dresselhaus, Phys. Rev. B 71, 205420 (2005).

${ }^{33}$ V. N. Popov, L. Henrard, and P. Lambin, Phys. Rev. B 72, 035436 (2005).

${ }^{34}$ V. N. Popov, L. Henrard, and P. Lambin, Nano Lett. 4, 1795 (2004).

${ }^{35}$ Z. Luo, L. Pfefferle, G. L. Haller, and F. Papadimitrakopoulos, J. Am. Chem. Soc. 128, 15511 (2006).

${ }^{36}$ R. Saito, G. Dresselhaus, and M. S. Dresselhaus, Phys. Rev. B 61, 2981 (2000). 